\title{
Estrategias didácticas en un entorno universitario como apoyo a la educación presencial
}

\section{Didactic strategies in a university environment as support for presence education}

\author{
NERI-VEGA, Jovita Georgina†, QUEZADA-MORENO, Maribel, CORTÉS-ÁLVAREZ, Yolanda y
} GONZÁLEZ-NERI, Aarón Iván

Universidad Autónoma de Querétaro, Facultad de Contaduría y Administración, Campus San Juan del Río

ID $1^{\text {er }}$ Autor: Jovita Georgina, Neri-Vega / ORC ID: 0000-0002-8435-3561, Researcher ID Thomson: X-2329-2018, CVU CONACYT ID: 497001

ID $1^{\text {er }}$ Coautor: Maribel, Quezada-Moreno

ID $2^{\text {do }}$ Coautor: Yolanda, Cortés-Álvarez / CVU CONACYT ID: 595702

ID $3^{\text {er }}$ Coautor: Aarón Iván, González-Neri / ORC ID: 0000-0002-4082-0746, CVU CONACYT ID: 503735

DOI: $10.35429 / \mathrm{JCP} .2019 .10 .3 .34 .46$

Recibido 30 de Agosto, 2019; Aceptado 31 de Diciembre, 2019

\begin{abstract}
Resumen
Se presenta en este trabajo una investigación relacionada con las estrategias de aprendizaje utilizadas en un entorno universitario en educación presencial, las estrategias que se mencionan son: la clase magistral activa, el estudio de casos, el debate, el juego de roles y el aprendizaje basado en proyectos. Metodología: Se utilizó un diseño descriptivo, llevando a cabo la recopilación de datos mediante el "Cuestionario de Evaluación de las Estrategias de Aprendizaje de los Estudiantes Universitarios". Resultados: El alumnado dispone de suficientes estrategias de aprendizaje para alcanzar el objetivo de sus estudios universitarios con éxito. Además, existe aceptación por parte del alumnado de la aplicación de las diversas estrategias en un curso presencial, ya que les permite abordar los temas utilizando la estrategia que permita un mejor aprendizaje. Conclusiones: Dado el proceso de aplicación del modelo educativo en la Educación Superior, deben crearse ambientes de aprendizaje que faciliten la innovación y participación del alumnado en el proceso de enseñanza-aprendizaje, considerando tanto el componente académico de la enseñanza como la dimensión humana. En el alumnado de la modalidad presencial, los resultados demuestran mejores habilidades de almacenamiento de la información.
\end{abstract}

Estrategias de aprendizaje, Alumnos, Educación Superior, Formación presencial

\begin{abstract}
This work presents research related to the learning strategies used in a university environment in face-to-face education, the strategies mentioned are the active master class, the case study, the debate, the game of roles and project-based learning. Methodology: A descriptive design was used, carrying out data collection through the "University Students Learning Strategy Assessment Questionnaire". Results: Students have enough learning strategies to achieve the objective of their university studies successfully. In addition, there is acceptance by students of the implementation of the various strategies in a face-to-face course, as it allows them to address the issues using the strategy that allows better learning. Conclusions: Given the process of applying the educational model in Higher Education, learning environments should be created that facilitate innovation and student participation in the teaching-learning process, considering both the component teaching as the human dimension. In in-person students, the results demonstrate better information storage skills.
\end{abstract}

Learning strategies, Students, Higher education, Faceto-face trainin

Citación: NERI-VEGA, Jovita Georgina, QUEZADA-MORENO, Maribel, CORTÉS-ÁLVAREZ, Yolanda y GONZÁLEZNERI, Aarón Iván. Estrategias didácticas en un entorno universitario como apoyo a la educación presencial. Revista de Pedagogía Crítica. 2019, 3-10: 34-46

$\dagger$ Investigador contribuyendo como primer autor. 


\section{Introducción}

Se presenta en este trabajo una investigación relacionada con las estrategias de aprendizaje utilizadas en un entorno universitario en educación presencial, las estrategias que se mencionan son: la clase magistral activa, el estudio de casos, el debate, el juego de roles y el aprendizaje basado en proyectos. Metodología: Se utilizó un diseño descriptivo, llevando a cabo la recopilación de datos mediante el "Cuestionario de Evaluación de las Estrategias de Aprendizaje de los Estudiantes Universitarios". Resultados: El alumnado dispone de suficientes estrategias de aprendizaje para alcanzar el objetivo de sus estudios universitarios con éxito.

Además, existe aceptación por parte del alumnado de la aplicación de las diversas estrategias en un curso presencial, ya que les permite abordar los temas utilizando la estrategia que permita un mejor aprendizaje. Conclusiones: Dado el proceso de aplicación del modelo educativo en la Educación Superior, deben crearse ambientes de aprendizaje que faciliten la innovación y participación del alumnado en el proceso de enseñanza-aprendizaje, considerando tanto el componente académico de la enseñanza como la dimensión humana. En el alumnado de la modalidad presencial, los resultados demuestran mejores habilidades de almacenamiento de la información.

\section{La clase magistral activa}

\section{Concepto}

La clase magistral activa es una exposición interactiva que consiste en la presentación clara y organizada de un tema para promover la comprensión y construcción del conocimiento por parte de los estudiantes.

El docente dedica tiempo de la clase al análisis de los contenidos, e involucra activamente a los estudiantes en el proceso de enseñanza aprendizaje.

Existen estudios realizados en ámbitos disciplinares diversos como la historia y la ingeniería que muestran que las clases magistrales activas contribuyen a generar interés, involucrar a los estudiantes y a proporcionarles estructura conceptual. (Morton 2009).

\section{Aprendizajes que promueve en los estudiantes}

La integración de los conocimientos sobre los temas tratados con situaciones de la realidad. Análisis y evaluación de problemas en los que tienen que aplicar los conceptos aprendidos. Comprensión profunda y clara de conceptos y estructuras teóricas claves para la actuación en situaciones académicas y profesionales

\section{Aplicación de la Clase magistral}

La fase de inicio es una oportunidad de generar interés formulando preguntas intelectualmente retadoras orientadas a identificar los saberes previos de los estudiantes. También, se puede presentar una situación cercana a los estudiantes que despierte motivación en torno al tema.

Es importante tomar en cuenta algunas condiciones para implementar una clase magistral activa según cada etapa de la sesión de clase. Durante el desarrollo de la clase, se presenta el contenido mientras se interactúa activamente con los estudiantes mediante la presentación de ejemplos, la formulación de preguntas y el fomento del diálogo. Se deben hacer pausas cada diez o quince minutos, en las que conviene realizar una recapitulación o un cambio de tema. Estas pausas son también una oportunidad valiosa para promover la construcción de conocimientos.

En el cierre de la sesión, es conveniente realizar, a un tiempo con los estudiantes, una síntesis de los temas trabajados en la sesión. Este momento de la clase también sirve para resolver dudas que quedaron pendientes durante el desarrollo del tema.

Un aspecto transversal a las tres etapas mencionadas es el uso de apoyos visuales como fotografías, mapas, videos, entre otros.

Aunque si bien no son imprescindibles, favorecen que el docente tenga una ruta clara durante la sesión, y brindan a los estudiantes un apoyo para la comprensión de los temas trabajados. Exley y Dennick (2004) señalan que pueden usarse tanto para apoyar la misma información que se presenta en la exposición, como para ampliarla. 
Esta herramienta, dependiendo de la asignatura permite a los profesores reflexionar y tomar en cuenta que primero tiene que conducir a los estudiantes a la comprensión de ciertos conocimientos. Por ello, antes de cada sesión, los estudiantes tienen que realizar lecturas previas que contribuirán a la participación en la clase. Ya que: el objetivo es que a través de la exposición interactiva los estudiantes establezcan comparaciones en términos de semejanzas y diferencias de los diferentes conceptos surgidos a lo largo del tiempo.

Algunos profesores han elegido la clase magistral activa por la naturaleza del curso que enseñan.

Al poner en práctica la clase magistral activa, se puede partir también de los intereses de los estudiantes y tomar en cuenta los rasgos generales de su perfil: jóvenes universitarios cuyas edades son similares y la carrera que estudian.

Si realmente todos tienen una actitud de contribuir como individuos al conocimiento grupal, a la misión, a la tarea, a la pregunta, pueden resultar cosas muy interesantes.

El intercambio exige autodisciplina, manejo de conocimientos y competencia profesional, y conduce al estudiante a colocarse en el papel que debe desempeñar como profesional de gestión.

Se dirige también a que el estudiante tome conciencia de que debe tomar decisiones no solo en el ámbito profesional sino también en el personal, de manera que se involucre y comprometa con la construcción de su propio conocimiento.

Las evaluaciones se dirigen a problematizar la teoría expuesta y plantear situaciones del ámbito profesional.

Las clases magistrales activas brindan a los estudiantes elementos para la comprensión de temáticas importantes en su formación.

Permiten organizar y dosificar mejor los contenidos, y asegurar que los estudiantes dispongan de toda la información necesaria para entender los contenidos.
La evaluación continua en una clase basada en exposiciones va desde la intervención en la sesión hasta la integración de saberes en el curso.

\section{El estudio de casos}

\section{Concepto}

El estudio de casos consiste en el análisis de una situación real o realista que presenta problemas y retos ante los cuales los estudiantes deben tomar decisiones fundamentadas en los enfoques o teorías del ámbito disciplinar o especialidad del curso en cuestión.

Un caso puede proporcionar a los estudiantes un entorno seguro en el cual actuar ante determinadas problemáticas, pero sin tener que asumir las consecuencias de eventuales errores. Se trata de una excelente forma de aprovechar el error del razonamiento, análisis o juicio de los estudiantes como una oportunidad de aprendizaje.

\section{Aprendizajes que se promueven}

El pensamiento crítico en situaciones complejas, Análisis, comprensión en interpretación de datos relevantes, reconocimiento y distinción de suposiciones de inferencias, formulación de juicios y toma de decisiones fundamentales, así como la presentación de ideas y opiniones ante diversos aspectos. Permite identificar y definir problemas propios del campo disciplinar. $\mathrm{Al}$ emplear el estudio de casos, el docente brinda oportunidades a los estudiantes que según Jonassen (2011) el análisis de casos es la forma por excelencia de promover que los estudiantes desarrollen una íntegra comprensión que les permita resolver problemas reales.

\section{Aplicación del estudio de casos}

El planteamiento del caso es el punto de partida. De acuerdo con Nilson (2010), un buen caso debe presentar con claridad la situación, los involucrados y sus intereses, y debe contar con las siguientes características:

a) Realismo: sea real o ficticio, el caso debe presentar una situación relevante propia de la carrera. Asimismo, el realismo hace posible que los estudiantes se sientan identificados con la situación. El presentar especificaciones técnicas, desarrollar personajes o presentar detalles históricos ayuda a darle realismo al caso. NERI-VEGA, Jovita Georgina, QUEZADA-MORENO, Maribel, CORTÉS-ÁLVAREZ, Yolanda y GONZÁLEZ-NERI, Aarón Iván. Estrategias didácticas en un entorno universitario como apoyo a la educación presencial. Revista de Pedagogía Crítica. 2019 
b) Incertidumbre: un buen caso permite distintas soluciones entre las cuales es válido el debate.

c) Riesgo: las decisiones que tomen los estudiantes impactarán, aunque sea de manera ficticia, en la salud, el bienestar o la supervivencia de una comunidad u organización. Es decir, se recomienda que algo valioso esté en juego.

El docente puede orientar la comprensión de los estudiantes a través de la formulación de preguntas como ¿cuál es el problema?, ¿qué soluciones posibles existen y cuáles serían las implicancias de implementarlas?, ¿cómo podría haberse evitado el problema? Etc.

En la fase final, es importante que el docente guíe a los estudiantes en el análisis de las implicancias de la vía de solución que propusieron, y que los ayude a que deduzcan si fue la alternativa más adecuada o no, lo que promueve el desarrollo de su juicio crítico.

En esta etapa, el trabajo de los estudiantes consiste en construir vías de solución del problema o problemas identificados.

Estas vías de solución deben estar fundamentadas en el conocimiento científico y disciplinar, en experiencias bien documentadas, o en el propio juicio de los estudiantes en la medida que estos puedan argumentar a favor de la solución que presenten.

Los casos propuestos en el curso están ligados a un plan de trabajo semestral. El docente utiliza un conjunto de casos que renueva periódicamente.

Para la selección toma en cuenta dos requisitos: el caso es vulnerable tanto al tiempo y al contexto temporal, como al espacio. De acuerdo con su experiencia, cuando no se toman en cuenta estos requisitos, no se logran los objetivos

Para promover el aprendizaje es necesario que los estudiantes distingan lo fundamental de lo accesorio. Trabajar un caso donde se resalta esa parte fundamental es la mejor plataforma de aprendizaje, porque permite conectar el aprendizaje conceptual-teórico con una aplicación concreta de la realidad.
El aprendizaje tiene mejores probabilidades de adquirir significado en la medida en que el estudiante puede identificar muy rápidamente una relación con la realidad, con lo que vive.

Los casos, una vez redactados y revisados, se ponen a disposición de los estudiantes con anticipación, muchas veces desde el comienzo del semestre. Cada sesión de clase está relacionada con un caso y ligada a un marco conceptual específico. Los estudiantes deben asistir a todas las clases realizando lecturas previas.

Para realizar el estudio de casos, los estudiantes tienen libertad para organizarse para trabajarlos individualmente $\mathrm{o}$ en pequeños grupos. En clase, según la interacción con los estudiantes, se revisa primero la parte conceptual, para observar en qué grado han logrado entender aquello que leyeron. La idea es que se construya y se alcance lo que el docente denomina homogenización del contenido, una comprensión común sobre estándares mínimos, que en algunas ocasiones se logran mejor que en otras, dependiendo la complejidad del tema. Una vez desarrollada esta fase, se pasa al estudio del caso: Esto que se ha comprendido de manera abstracta, se va a aterrizar de manera concreta. El caso funciona entonces a juicio del docente como una plataforma de aterrizaje.

La evaluación del curso puede consistir en un $90 \%$ en resolver casos. Si bien estos pueden variar en contenido y ser distintos a los que han servido para desarrollar la sesión de clase, el objetivo es el mismo. Los estudiantes ya vienen mentalizados, que el aprendizaje tiene, dos grandes fuentes, lo que ellos son capaces de leer, los textos que se les recomienda, y lo que van a encontrar como plataforma de aterrizaje en los casos.

También se les debe explicar que para cada sesión de clase van a leer previamente sobre los aspectos conceptuales de los temas para luego compartir las lecturas con sus compañeros de grupo. 


\section{El Debate}

El debate es una estrategia de enseñanza aprendizaje que consiste en la confrontación de distintos puntos de vista con la finalidad de llegar a un juicio sólidamente sustentado. Cattani, 2003, define el debate como "una competición (un reto, un desafío) en la que, a diferencia de lo que ocurre en una simple discusión, existe una tercera parte (un juez, un auditorio) cuya aprobación buscan los dos contendientes".

Otra manera de formular el debate es realizarlo de un modo semiestructurado, en el cual se desarrolla una discusión controversial en el aula. Esta consiste en involucrar a los estudiantes en la reflexión y análisis sobre una pregunta polémica. A diferencia del debate que se ha detallado en el párrafo anterior, la discusión en aula promueve la participación libre y espontánea, y no necesariamente culmina con la identificación de un mejor argumento.

\section{Aprendizajes que se promueven}

Facilita el desarrollo de habilidades de análisis, interpretación y síntesis de información, lo cual conduce a establecer relaciones entre distintos temas.

Fomenta las habilidades de investigación como la identificación y procesamiento de la información de diversas fuentes; la organización de información acerca de un tema o problema o la revisión de los planteamientos con el propósito de dar sustento a sus argumentos.

Permite, respecto a la dimensión actitudinal y valorativa, el reconocimiento de la diversidad de visiones sobre los problemas sociales y el respeto de diferentes puntos de vista.

Promueve el desarrollo de habilidades comunicativas como la exposición de opiniones, sentimientos, ideas y experiencias de manera fundamentada y coherente.

\section{Aplicación del Debate}

El docente, teniendo en cuenta una temática que sea pertinente al curso, redacta una proposición ante la cual los estudiantes deben tomar posición.
Huber y Snider (2006), autores de un texto que influyó mucho en el uso educativo del debate, señalan que la proposición puesta a debate debe tener algunas características deseables:

a) Formulación de una proposición debatible: La ruta para trabajar un debate en el aula universitaria puede ser la siguiente:

El docente presenta a los estudiantes la proposición a debatir, y establece el formato de trabajo. En un debate estructurado, se especifica cuántos equipos habrá y cómo estarán constituidos, el tiempo del que dispondrán para la etapa preparatoria, la composición del jurado (es posible que el único juez sea el docente, pero también se podría conformar un jurado con otros docentes o con estudiantes), y los criterios con los cuales el juez evaluará el desempeño de los equipos.

Con la finalidad de que el debate realmente cumpla un cometido pedagógico, los estudiantes realizan un ejercicio de análisis y comprensión del resultado interesante para el debatiente.

El Debate, provoca desacuerdo, se puede apoyar en evidencias, merece la pena discutirla, se pueden comparar valores, es una sola cuestión, es clara, es actual o actualizada, es oportuna, se puede cubrir en el tiempo disponible, es adecuada para una presentación oral.

b) Preparación de las condiciones: proposición, para lo cual, si es necesario, han de consultar a expertos o buscar bibliografía. Después es momento de pensar argumentos a favor y en contra de esta, independientemente de la postura que ellos tengan que asumir en el debate.

A continuación, los estudiantes empiezan la fase de investigación, para identificar la evidencia que respalde la postura que defenderá el equipo. Sobre la base de información identificada y organizada, cada equipo debe construir una tesis, una toma de posición fundamentada ante la proposición en debate. Es importante que la tesis se formule de forma escrita pero que también se practique y ensaye oralmente antes del debate. 
Además de las pautas formales detalladas, el docente puede fomentar un debate a modo de una discusión abierta en aula en la cual los estudiantes exponen diversos puntos de vista en relación con un tema de manera más espontánea.

c) Desarrollo del debate argumentación reunión de equipos réplica o refutación: Los equipos exponen la postura que defenderán según el sorteo realizado con anterioridad. Cada equipo presenta su exposición siguiendo una estructura básica: introducción, desarrollo y conclusión. Los miembros de cada equipo utilizan sus habilidades comunicativas y críticas para discutir los argumentos contrarios, y posicionar los argumentos propios ante el público y jurado que los escucha. Los equipos disponen de un tiempo asignado previamente. Cada equipo debe preparar una contraargumentación. Para ello, es importante que realice un análisis de su propia información, así como de la postura contraria.

d) El debate cierra con la calificación del jurado. El docente puede decidir que el resultado del debate influya en la calificación del estudiante. Cabe recordar que la experiencia y la investigación muestran que los estudiantes se involucran más en actividades que saben que serán tomadas en cuenta para su calificación. Si el docente opta por hacer una discusión en aula, es importante considerar que la naturaleza de una discusión controversial involucra respetar el ritmo y el rumbo a veces impredecible al que puede llevar el tema. Es importante que el docente busque generar discusiones activas y participativas en las cuales se involucren todos o la mayor parte de los estudiantes.

En la realización del debate, el docente evalúa el razonamiento que ha seguido el estudiante para determinar su respuesta o cuáles son los argumentos que tiene para poder enfrentar determinada situación problemática. Luego, se retroalimenta para que los estudiantes mejoren en su desempeño. En este proceso se pone énfasis en que no se pretende que el ejercicio sea perfecto, sino que sirva para mejorar su desempeño en el curso y en el ámbito profesional. El último debate suele ser el mejor, como consecuencia del aprendizaje logrado de sus demás compañeros.
Asimismo, la retroalimentación se realiza en un clima de confianza y, respeto y aprecio hacia los estudiantes, lo importante es corregir para, dado el caso que les toque en el ejercicio profesional una situación en la que se equivoquen, poder salir también rápidamente. En el examen final se evalúa la capacidad del alumno de poder argumentar adecuadamente, persuadiendo sobre determinada posición.

Para la realización del debate, el docente comenta las indicaciones que deben seguir los estudiantes durante su desarrollo. Se les pide que vengan a hacer la defensa de una posición y no una exposición, de modo tal que el verbo que tienen que traer es el verbo persuadir. Tienen que venir a convencer. Una vez terminado el debate entre los grupos, se invita a la audiencia a participar formulando preguntas, algunos temas son más polémicos que otros, algunas clases estudiantes están más incentivados que otros. El profesor hace un comentario final donde a cada uno de los alumnos se les dice en qué cosa ha fallado y en qué cosas ha sido positivo.

Las discusiones que promueve el docente se convierten entonces en entrenamientos que permiten ejercitar el razonamiento, porque el alumno va dando su opinión, entonces se le va orientando en la ilación que hay detrás de su razonamiento y en la información que utiliza para llegar a esa conclusión. La orientación permanente durante este proceso hace posible que el estudiante agudice su razonamiento y no se limite al almacenamiento de conocimientos.

A través de las discusiones, el docente conduce a los estudiantes a integrar lo aprendido. Durante el intercambio de ideas, toma en cuenta también las inquietudes e intereses de los estudiantes.

La retroalimentación del docente durante el desarrollo del debate o la discusión cumple la función de mejorar el desempeño de los estudiantes. El debate se complementa con diversas actividades orientadas al aprendizaje como exposiciones y análisis de casos. Los estudiantes intervienen en el debate o en la discusión conociendo las posiciones en favor y contra, lo que conduce a la elaboración de argumentos sólidos y consistentes para defender su postura. 


\section{Juego de roles}

El juego de roles es una estrategia que permite que los estudiantes asuman y representen roles en el contexto de situaciones reales o realistas propias del mundo académico o profesional (Martín, 1992). Es una forma de llevar la realidad al aula.

$\mathrm{Si}$ bien en un juego de roles los estudiantes deben ajustarse a reglas, tienen libertad para actuar y tomar decisiones, de acuerdo con cómo interpretan las creencias, actitudes y valores del personaje que representan. A diferencia de otro tipo de simulaciones y dramatizaciones, en el juego de roles se establecen las condiciones y reglas, pero no existe un guion predeterminado.

\section{Aprendizajes que se promueven}

Desarrollar competencias profesionales y académicas propias de la carrera o especialidad. Reconocer formas alternativas de pensar y actuar. Desarrollar relaciones interpersonales y habilidades de comunicación. Facilitar la transferencia del aprendizaje hacia situaciones de la vida profesional. Estimular que los estudiantes exploren y comprendan su propio comportamiento y el de otras personas en situaciones simuladas. Promover un proceso de auto análisis del rol asumido a partir de la retroalimentación brindada.

\section{Aplicación del juego de roles}

El docente presenta a los estudiantes la situación o contexto en la cual se desenvolverá el juego. Es importante señalar las condiciones que caracterizan a la situación, distinguiendo aquellas condiciones que los personajes pueden cambiar aquellas sobre las cuales no tienen influencia. Además, el docente debe determinar los actores y sus características, cuidando de establecer sus concepciones, valores e intereses en la situación. También, requiere establecer el poder y los recursos que tienen los distintos actores, y presentar las reglas de juego. En esta fase, es importante no perder de vista cuáles son las competencias que buscamos que los estudiantes desarrollen, de modo que los elementos de la situación contribuyan al desarrollo de estas.
El proceso de implementación del juego de roles puede seguir el siguiente curso: Una vez presentada la situación, es importante establecer quiénes representarán roles y quiénes participarán como espectadores. Se les brinda un tiempo para analizar la situación y adaptarse a ella $y$ al papel que han de representar preferentemente, de ser posible, en un espacio aparte. Es importante Después de la fase de juego, se analiza lo que sucedió, habitualmente en una plenaria. Es importante poner en común nuevamente cuál era el problema o reto que afrontaban los protagonistas del juego.

Sobre esa base, el docente invita a los estudiantes a que opinen de manera fundamentada sobre las acciones y decisiones que tomaron los distintos personajes, y sobre el desenlace o resultado logrado. También podría preparar una guía de preguntas que orienten la discusión. El cierre del juego es también el momento en el cual el docente guía a los estudiantes a establecer relaciones significativas entre lo que sucedió en el juego y la realidad.

En esta fase, es importante que el docente recuerde que quien representa un personaje debe pensar, actuar y decidir como lo haría la persona a la que representa. El docente o un estudiante designado cuidará que la representación se realice en los tiempos previstos.

a) Organización de la clase para el juego cierre del juego desarrollo del juego: Comunicar a los estudiantes que el juego de roles no es solo representar un papel, sino sentirse dentro de él. Por otro lado, es importante que los estudiantes que participaran como observadores analicen la situación sobre la base de criterios y con la guía docente.

El proceso de cómo el estudiante forma parte de un juego de roles tiene varias fases. Previamente al primer juego de rol, el docente explica a los estudiantes los cuatro principios fundamentales para que un abogado pueda persuadir con el lenguaje.

Estos cuatros principios son: el manejo de contenido, el manejo del lenguaje verbal, el manejo del lenguaje no verbal y la capacidad de poder establecer algún nivel de conocimiento con la audiencia, en este caso la audiencia son jueces o árbitros. 
El juego de roles permite colocar al estudiante, poco a poco en la situación real y vivir la experiencia de experimentar distintos roles relacionados con el ejercicio de su profesión.

Esta experiencia inicial permite dialogar con el estudiante sobre cómo se sintió en el rol, qué tanto se metió en el papel y si se convenció de su desempeño.

Posteriormente, se le solicita al estudiante que exponga frente a un grupo arbitral. La exposición incluye los hechos y el desarrollo de fondo del caso. Terminada esta segunda etapa, se pasa a una tercera en la cual se acerca aún más al estudiante a una situación real. En este caso, son los jueces o los árbitros quienes comienzan a formular preguntas. El expositor debe ser persuasivo al responder.

La evaluación del curso es sobre el juego de roles. Los que se desarrollan durante las clases pueden ser calificados o no, en función a cómo el docente acuerde con quien funge como árbitro o jurado. Pero lo que siempre se cumple es que la evaluación para la calificación final siempre es un juego de roles. Esta evaluación se desarrolla en un salón ambientado para simular las condiciones reales y los estudiantes tienen que ir vestidos y presentarse tal como lo harían en un escenario real.

El juego de roles contiene una metodología adecuada para ilustrar una situación típica que los estudiantes encontrarán en una organización que estará pronta a implementar un nuevo sistema. Los estudiantes tienen la oportunidad de ponerse en el lugar de cada parte.

\section{Aprendizaje Basado en Proyectos}

El aprendizaje basado en proyectos es una metodología que se desarrolla de manera colaborativa que enfrenta a los estudiantes a situaciones que los lleven a plantear propuestas ante determinada problemática. Se entiende como un proyecto el conjunto de actividades articuladas entre sí, con el fin de generar productos, servicios o comprensiones capaces de resolver problemas, o satisfacer necesidades e inquietudes, considerando los recursos y el tiempo asignado.
Los autores e investigadores que proponen los modelos por competencias en la educación consideran que el proyecto es una estrategia integradora por excelencia, y que es la más adecuada para movilizar saberes en situación. (Díaz Barriga 2015; Jonnaert et. al. 2006). De esta manera, los estudiantes pueden planear, implementar y evaluar actividades con fines que tienen aplicación en el mundo real más allá del salón de clase.

\section{Aprendizajes que se promueven}

Negociar compromisos y tomar decisiones, plantear soluciones y generar ideas innovadoras, evaluar en conjunto la organización y avance del equipo, escuchar a los compañeros del equipo y emitir sus puntos de vista, planificar el trabajo en equipo para el logro de metas comunes.

\section{Aplicación del Aprendizaje basado en proyectos}

En esta etapa, los estudiantes reconocen una situación relevante vinculada a uno de los temas del curso que requiere ser trabajada a través de un proyecto. Generalmente, el docente presenta algunas propuestas para que los estudiantes seleccionen aquella que más les interese. Luego, se debe estimular la motivación y el entusiasmo en la tarea, por ejemplo, señalando la importancia e impacto del proyecto, compartiendo experiencias profesionales, presentando información de investigaciones o proyectos anteriores y formulando preguntas retadoras.

Asimismo, un aspecto muy importante es la organización del equipo y la distribución de responsabilidades. Si bien es recomendable que cada equipo se organice de manera autónoma, el docente debe orientar las dinámicas internas e intervenir cuando lo considere necesario. También se requiere que acompañe al equipo en la generación de ideas para asegurar que los proyectos tengan una clara dirección y sustento.

a) Planteamiento del proyecto y organización: La implementación exitosa de un proyecto requiere que en el proceso esté presente una característica básica, una pregunta o concepto central en el curso, sobre la cual los estudiantes han de investigar. En el proceso, se pueden identificar las siguientes etapas. 
Con el propósito de conocer el tema del proyecto y profundizar en los fundamentos de este, Donnelly y Fitzmaurice (2005) recomiendan que los estudiantes recojan información. Se sugiere brindar constantemente una retroalimentación a cada uno de los equipos con ayuda de preguntas guía a fin de que enfoquen de manera pertinente su investigación con el proyecto que llevarán a cabo.

El proyecto tiene como finalidad generar un producto, un servicio o brindar una experiencia. En esa línea se espera que los estudiantes construyan los aprendizajes durante el proceso y que estos tengan una relación directa con los resultados de aprendizaje del curso. En esta segunda etapa, los estudiantes establecerán los objetivos, considerando los temas principales del curso, los recursos con los que cuentan y los tiempos. Asimismo, se elabora un listado de las actividades necesarias para lograr los objetivos del proyecto.

De acuerdo con la naturaleza y dificultad del curso y del proyecto escogido, y a las características de la clase, los estudiantes necesitarán mayor o menor monitoreo en el proceso. Es necesario que el docente esté atento a las dificultades y finalmente se presentan los productos desarrollados por los estudiantes y se exponen los resultados del proyecto, lo cual requiere preparación previa que permitirá el despliegue de competencias tales como la comunicación oral y escrita. Para esta etapa, se recomienda contar con criterios claros que sirvan de guía a los equipos, promover la autoevaluación de cada uno de los miembros del equipo y la evaluación entre pares.

También, es recomendable reservar un momento para evaluar en plenario cómo se sintieron a lo largo del proceso y cuáles han sido las lecciones aprendidas. Es decir, el proyecto cierra con una evaluación tanto de lo logrado con el proyecto como de lo aprendido por los estudiantes.

b) Investigación sobre el tema, Definición de los objetivos y plan de trabajo, Implementación, Presentación y evaluación de los resultados: Las oportunidades que vayan surgiendo, que orienten a los estudiantes en caso de que necesiten reajustes en su plan y que soliciten productos intermedios durante el proyecto, que sean calificados y que aseguren el éxito de la presentación final.
Seleccionar el tema del proyecto. En lo posible, el tema del proyecto debe partir de algo que los estudiantes vean muy cercano a ellos, que tenga una utilidad o que despierte su interés en gran medida, y, sobre todo, que permita aplicar los conocimientos desarrollados en clase y, a la vez, desarrolle nueva información. En esa búsqueda se involucra a los estudiantes, de manera que se genere un mayor interés por el proyecto.

Se debe comunicar a los estudiantes lo que se pretende desarrollar en el proyecto y lo que se requiere para la presentación de este. Para ello se les presenta un documento al inicio del ciclo que pueden revisar permanentemente. Asimismo, se genera un mayor interés cuando se explicita que el proyecto tendrá un impacto dentro o fuera de la universidad. Por esa razón, se requiere explicar de qué manera se darán a conocer las aportaciones de cada uno de los proyectos.

Evaluar vías alternativas de presentación. $\mathrm{Si}$ no se dispone de mucho tiempo para desarrollar una sesión presencial de evaluación, los estudiantes pueden elaborar un video de su proyecto y compartirlo dentro de un grupo cerrado de discusión. Este ofrece la ventaja de que el proyecto pueda ser revisado por jurados en distintos lugares y de acuerdo con su disponibilidad.

Retroalimentar al estudiante. El estudiante debe recibir una adecuada retroalimentación con relación a la calificación de su proyecto y los aspectos de este. Es importante que evidencie que el proyecto ha sido detalladamente revisado.

Los estudiantes tienen diversas tareas como parte de su carga académica regular, por lo que, si se quiere desarrollar un buen proyecto, es necesario brindarles flexibilidad en las fechas de entrega, así como acordar con ellos la presentación de avances a lo largo del ciclo que les permita distribuir mejor sus tiempos y responsabilidades al interior del grupo.

Mantener una vía de asesoría y motivación permanente. El docente brinda asesoría y motivación, tanto presencial como virtual, durante todo el tiempo de preparación del proyecto. 
Esto permite que proporcione herramientas diversas para el desarrollo del proyecto como, por ejemplo, sitios web de información relacionada al tema, recursos bibliográficos, contactos con profesionales expertos en el tema, recursos que ayuden a mejorar la presentación del proyecto, entre otras. La tecnología, actualmente, ofrece la oportunidad de compartir con los estudiantes información a través, por ejemplo, de las redes sociales, específicamente mediante un grupo de Facebook. (Sánchez, García, Steffens y Palma, 2019). Puede ser adecuado que no solo sea el profesor del curso el que evalúe el proyecto. Se puede conformar un jurado con especialistas en el tema, profesores de otros cursos, o, en general, personas relacionadas al tema.

La investigación sobre la problemática específica y la reflexión acerca de cómo esta se articula con dichos enfoques. Que el acompañamiento del proceso de diseño del proyecto y de la implementación de las actividades sea permanente.

Esto ayudará a que los estudiantes puedan organizar su trabajo de manera eficaz y eficiente, y que en dicho proceso puedan reflexionar sobre las capacidades en juego. Algo que puede resultar útil en el curso es brindar tiempo en la misma sesión de práctica para que, con la asesoría de los profesores, los grupos puedan realizar algunas tareas específicas.

En segundo lugar, para aportar al proceso reflexivo sobre la problemática y su relevancia social se busca que la dimensión operativa del proyecto no impida que se pierdan de vista los sentidos y objetivos sociales detrás de la acción. En esta línea, en el proceso de diseño e implementación del proyecto se incluyen momentos de presentación y reflexión colectiva sobre los avances. Además, al final del proceso (y del curso) se dedica una sesión completa de práctica donde los estudiantes evalúan las acciones implementadas.

En tercer lugar, se debe brindar contención subjetiva con respecto al tratamiento de la temática abordada. En la mayoría de los casos, los problemas que los estudiantes abordan los interpelan sobre sus propias subjetividades, historias personales y el entorno en el que se encuentran, y en tal sentido, es importante acompañarlos en la elaboración de dichos procesos.
Promover que los estudiantes, desde el inicio, se perciban como protagonistas del proceso y agentes capaces de generar impacto en su entorno. La idea es fomentar que seleccionen las temáticas, se involucren e investiguen sobre ellas, para luego discutirlas. En un segundo momento, se motiva para que la temática general a tratar, la puedan aterrizar en un caso y contexto específico de su interés.

A lo largo del semestre deben pautarse presentaciones, entregables y actividades concretas que permitan analizar el avance de los grupos. Se debe diferenciar el trabajo y esfuerzo colectivo del individual. Si bien la dinámica de proyectos realza el trabajo en equipo, es importante que a lo largo del proceso se tenga presente la evaluación individual. El docente durante el proceso cumple un papel fundamental como orientador y guía que acompaña a sus estudiantes en la construcción de su propio conocimiento.

\section{Metodología}

Se utilizó un diseño descriptivo, llevando a cabo la recopilación de datos mediante el Cuestionario de Evaluación de las Estrategias de Aprendizaje de los Estudiantes Universitarios.

El cuestionario se aplicó a un grupo de $4^{\circ}$ semestre de la licenciatura en Contador Público, del semestre 2019-1, a cuarenta estudiantes, donde se explicó los conceptos de cada una de las herramientas y posteriormente, cada alumno especificó los porcentajes de utilización de cada herramienta por cada uno de los profesores, dependiendo la asignatura que imparten. El cuestionario se formuló a manera de rúbrica, donde se dio espacio para algún comentario, el cuál se unificó y redactó de acuerdo con el más mencionado.

\section{Resultados}

\section{La Clase Magistral Activa}

En esta dinámica establecida por el docente, como cada presentación tiene que ir acompañada de una lectura previa, se generan una serie de discusiones que permiten el intercambio de ideas con el estudiante, las que lo conducen a identificar las motivaciones y las razones de fondo que dieron lugar al surgimiento de una conclusión en particular. 
Ninguna conclusión, ni ningún fenómeno del conocimiento surge espontáneamente, sino que es producto de algo. Entonces los estudiantes terminan haciendo esa conexión, finalmente desde la historia, desde el modelo mismo, desde las estrategias que ese conocimiento tuvo a mano para sacar adelante la propuesta.

\section{Estudio de casos}

Busca que los estudiantes construyan su propio conocimiento y le otorguen utilidad a lo aprendido: $\mathrm{Si}$ se logra captar eso (lo fundamental), el aprendizaje tiene mejores probabilidades de dejar un significado para el alumno, es decir, saberse responder a sí mismo la pregunta: esto que he aprendido, sirve para tal cosa.

El trabajar con el estudio de casos encierra el riesgo de que los estudiantes dejen de lado el campo teórico para concentrarse en el desarrollo del caso, sin haber rescatado los conceptos fundamentales. Por ello, se deben aplicar controles de lectura en las prácticas dirigidas que permiten asegurar que los estudiantes manejen la teoría.

El estudio de casos permite establecer conexiones entre la teoría y su aplicación concreta en la realidad. El estudiante debe hacerse responsable de revisar la teoría antes de cada clase para estar en condiciones de participar de la resolución del caso.

El docente construye casos, tomando en cuenta que deben ser actuales y cercanos y pertenecer al contexto del estudiante. El docente da indicaciones claras sobre las acciones que deben realizar los estudiantes y formula preguntas para orientar la resolución del caso. El estudio de casos se puede realizar de manera expositiva participativa, de manera colaborativa o individual.

\section{El Debate}

El docente pone en marcha el debate desde el inicio del ciclo. Al principio establece cuáles van a ser los temas más problemáticos de todo el curso y respecto a esos temas se programan los debates. Para ello divide el salón en parejas, y las agrupa de modo tal que en cada debate participen cuatro alumnos.
Dos defienden la posición A, y dos defienden la posición B. Por ejemplo, en un semestre que tenga 40 estudiantes, se pueden elegir diez temas problema a lo largo de todo el curso.

Cada tema está enunciado como una pregunta, a partir de la cual es posible responder con dos alternativas, que marcan una posición específica del tema seleccionado, estas posiciones se defienden. Previo al debate, los alumnos tienen que investigar sobre ese tema y distinguir los argumentos de las posturas a favor y en contra de su posición.

El docente busca profundizar en los temas y el desarrollo de habilidades necesarias para el desempeño profesional. El docente crea un clima de confianza como condición básica para que el debate o la discusión se lleven a cabo adecuadamente.

Selecciona temas polémicos del curso y formula preguntas construidas previamente que permitan confrontar dos posiciones opuestas.

\section{El juego de roles}

El juego de roles permite que el estudiante se imagine en una situación real y se desenvuelva asumiendo roles que deberá desempeñar como futuro profesional.

El docente valora los logros de los estudiantes en función al esfuerzo que realiza cada uno de ellos, lo cual contribuye al desarrollo de su estima y autonomía en el aprendizaje.

El docente da indicaciones claras sobre los principios que rigen el juego de roles, de manera que el estudiante conoce lo que se espera de el a través de su participación en esta dinámica.

\section{Aprendizaje Basado en Proyectos}

La implementación del proyecto permite el desarrollo de competencias importantes para el futuro desempeño profesional de sus estudiantes, como, por ejemplo, comunicación oral, o trabajo en equipo y toma de decisiones. Incluso esta metodología puede generar aprendizajes no esperados, como la persuasión o la negociación. 
En el desarrollo de sus proyectos, los estudiantes cuentan con enfoques o marcos de referencia teóricos claros, que les permitan poder aproximarse a las problemáticas con herramientas suficientes para poder profundizar en el análisis de estas y desarrollar sus propias reflexiones. En tal sentido, un aspecto fundamental del curso es la complementariedad entre las sesiones de clase y las sesiones de práctica. Por ejemplo, en las primeras semanas del curso, se desarrollan los enfoques y marcos de referencia teóricos en clases y esto coincide con la primera etapa del proceso de diseño.

Es recomendable que los estudiantes desde el primer día de clases formen grupos de cuatro participantes y durante todo el semestre estos grupos se mantengan, de esta manera la confianza entre ellos aumenta y esto es fundamental para poder trabajar en forma conjunta.

\section{Cuestionario de Evaluación de las estrategias de aprendizaje}

De las siguientes estrategias de aprendizajes, cuáles utiliza el profesor en la materia que imparte.

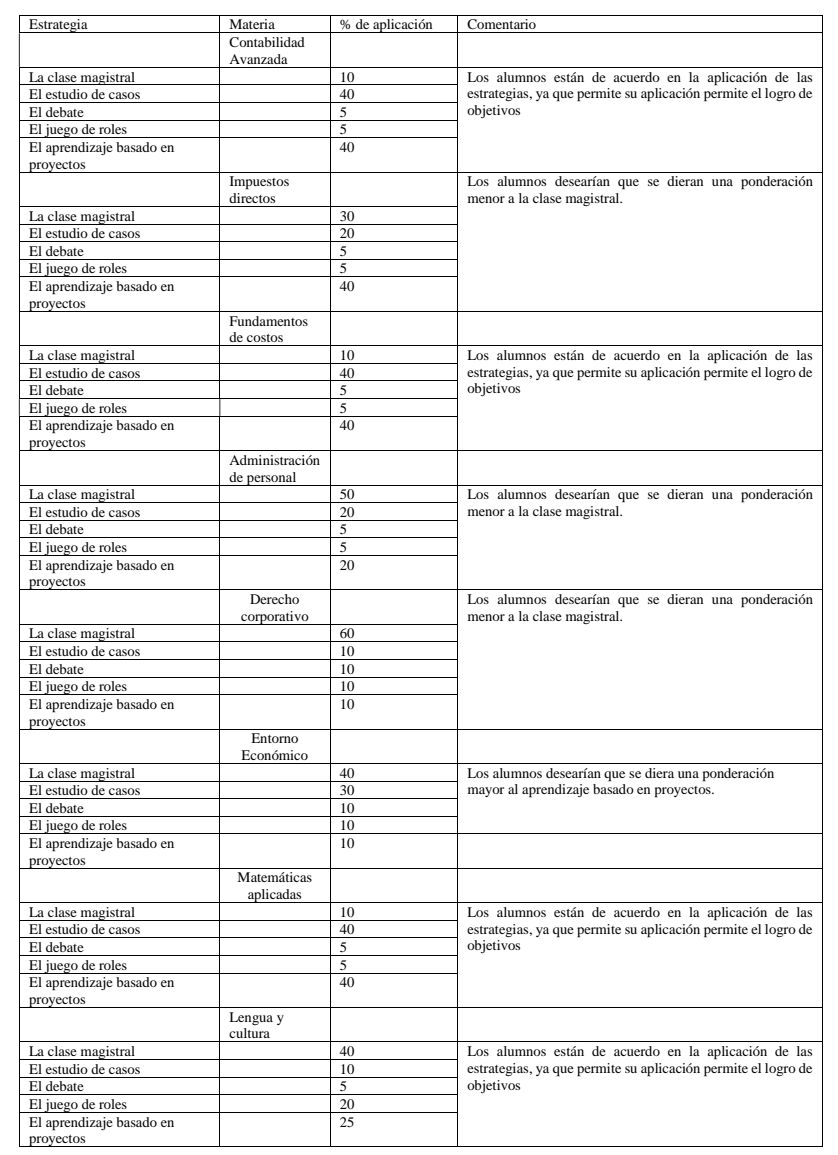

Tabla 1

Fuente: Elaboración Propia

\section{Conclusiones}

Dado el proceso de aplicación del modelo educativo en la Educación Superior, deben crearse ambientes de aprendizaje que faciliten la innovación y participación del alumnado en el proceso de enseñanza-aprendizaje, considerando tanto el componente académico de la enseñanza como la dimensión humana. En el alumnado de la modalidad presencial, los resultados demuestran mejores habilidades de almacenamiento de la información.

Se debe tener claridad sobre cuáles son los principales criterios que se buscan evaluar según los objetivos del curso, asociados a las competencias generales o específicas que se espera que el curso aporte.

La implementación de cada herramienta ha permitido al docente el desarrollo de competencias vinculadas con el curso. La implementación del proyecto permite integrar diversas áreas al manejo de los conocimientos relacionados con el curso.

La evaluación adecuada de cada herramienta permite que los estudiantes muestren los logros alcanzados en su elaboración, como lo aprendido por el estudiante. Asimismo, facilita la retroalimentación.

Cada herramienta, permite una evaluación dependiendo de la materia, y de esta manera la aplicación de cada herramienta ayuda al docente a realizar evaluaciones centradas en el aprendizaje significativo sin importar la herramienta aplicada.

Por otro lado, se brindan a los grupos la posibilidad de presentar públicamente alguna de las actividades diseñadas en sus proyectos lo cual tiene un efecto muy importante en su aprendizaje. Ello les da la posibilidad de interactuar con la comunidad universitaria, presentar sus reflexiones y propuestas, y evidenciar en la práctica su propia capacidad como agentes sociales.

Es importante que, iniciando el semestre, los estudiantes cuenten con la información suficiente que les permita tener claridad sobre los alcances de sus trabajos, las expectativas del curso con respecto a su trabajo, así como las fechas de los avances y entregas que deben ir desarrollando.

NERI-VEGA, Jovita Georgina, QUEZADA-MORENO, Maribel, CORTÉS-ÁLVAREZ, Yolanda y GONZÁLEZ-NERI, Aarón Iván. Estrategias didácticas en un entorno universitario como apoyo a la educación presencial. Revista de Pedagogía Crítica. 2019 
Con los resultados obtenidos se puede concluir que, en las materias teóricas, los docentes utilizan más herramientas como la clase magistral activa, mientras que, en las materias prácticas, los docentes utilizan más el estudio de casos o bien el aprendizaje basado en proyectos.

\section{Referencias}

Cattani, A. 2003. Los usos de la retórica. Madrid: Alianza Ensayo.

Díaz, F. 2015. Estrategias para el desarrollo de competencias en educación superior. En: Carrillo, Gabriela (Ed). I Encuentro Internacional Universitario. El currículo por competencias en la educación superior. Ponencias y debate. Lima. PUCP.

Donnelly, R. y Fitzmaurice, M. 2005 Collaborative Project-based Learning and Problem-based Learning in Higher Education: a Consideration of Tutor and Student Role in Learner-Focused Strategies. En: O’NEILL, Geraldine,

Exley, K. y Dennick, R. 2004. Giving a Lecture. From Presenting to Teaching. Routledge Falmer.

Flores, F. y Ortiz, M. (2019). El conocimiento didáctico-tecnológico del contenido (CDTC) en docentes universitarios. Imágenes y modos de expresión en las prácticas áulicas. Revista del Instituto de Investigaciones en Educación, (11), 6-24.

Huber, R. y Snider, A. 2006. Influencing through argument. Updated Edition. Nueva York: International Debate Education Association.

Jonassen, D. 2011. Learning to Solve Problems: A Handbook for Designing Problem-Solving Learning Environments. New York: Routledge.

Martín, X. 1992. El role-playing, una técnica para facilitar la empatía y la perspectiva social. Comunicación, Lenguaje y Educación, número 15, pp. 63-67.

Morton, A. 2009. Lecturing for large groups. En: A Handbook for Teaching and Learning in Higher Education: Enhancing Academic Practice. Third Edition.
Nilson, L. 2010. Teaching at its best: a researchbased resource for college instructors. San Francisco: Jossey-Bass.

Sánchez, M. García, J. Steffens, E. Palma, H. (2019). Estrategias Pedagógicas en Procesos de Enseñanza y Aprendizaje en la Educación Superior incluyendo Tecnologías de la Información y las Comunicaciones. Información tecnológica, 30(3), 277-286. 